\title{
Optimization of the Hewlett-Packard Particle-Beam Liquid Chromatography-Mass Spectrometry Interface by Statistical Experimental Design
}

\author{
S. K. Huang and N. R. Garza \\ Hoechst Celanese Corporation, 1901 Clarkwood Road, Corpus Christi, Texas, USA
}

Optimization of both sensitivity and ionization softness for the Hewlett-Packard particle-beam liquid chromatography-mass spectrometry interface has been achieved by using a statistical experimental design with response surface modeling. Conditions for both optimized sensitivity and ionization softness were found to occur at $55-\mathrm{lb} / \mathrm{in}^{2}$ nebulizer flow, $35^{\circ} \mathrm{C}$ desolvation chamber temperature with approximately $45 \%$ organic modifier in the presence of $0.02-\mathrm{F}$ ammonium acetate and a liquid chromatography flow rate of $0.2 \mathrm{~mL} / \mathrm{min}$. ( $\mathrm{J} A \mathrm{~m}$ Soc Mass Spectrom 1995, 6, 507-512)

$Q^{\prime}$ ensitivity and ionization softness measure the effectiveness of particle beam liquid chromatography-mass spectrometry (LC/MS) interfaces. Sensitivity is determined in part by the efficiency with which the particle beam delivers the solute into the ionization chamber, whereas ionization softness is a measure of the relative ease of formation of molecular ions with respect to fragment ions subsequent to ionization of the particle beam.

Many variables [1, 2] influence solute delivery. These variables include mobile phase composition and flow rate, the extent of addition of a volatile salt into the mobile phase [3], the position of the fused silica tube in the desolvation chamber, the choice of nebulizer and its flow pressure, the pumping capacity, the size of the desolvation chamber and its temperature, the size and material of the skimmers in the momentum separator, the ion source temperature, and the nature of the compounds to be analyzed.

Normally, the optimization procedure involves adjustment of each factor independently and repeatedly until a perceived optimum is reached. As with all optimization schemes, this approach is subject to a false optimum [4]. Moreover, one may never reach the true optimum by using the "one variable at a time" approach if the variables interact $[4,5]$. Several methods to optimize a process include simplex algorithms and experimental design $[4,5]$. A design experiment is used to help understand the process and map through mathematical models the relationships among the variables within an operating space. Experimental designs,

Address reprint requests to $S$. K. Huang, Analytical Department, Hoechst Celanese Corporation, 1901 Clarkwood Road, Corpus Christi, TX 78469. in particular statistical experimental designs, are used to achieve one or several of the following goals:

1. Obtain the desired information and use the least resource, that is, the fewest experimental runs.

2. Determine the important variables or factors among many possible factors.

3. Take into account the natural variation of the data or experimental errors.

4. Identify the outliers and study their causes.

5. Determine whether the noise is statistically important.

6. Optimize the experimental system.

Statistical experimental designs have been used extensively in the manufacturing industries to optimize the yield, color, and hardness of the products as well as to minimize the emission of the undesired pollutants. For a good start, readers will find the ref. [6] interesting to read. Herein, the use of a set of designed experiments to help understand how the particle-beam LC/MS interface works, to map the relationship among the variables, and finally to determine optimum conditions is described.

Prior to running the experiments, all of the known variables were considered and evaluated for appropriateness for inclusion in the model. For the sake of simplicity, however, some of the variables that pertain to the interface design characteristics were held constant. These include the pumping capacity, the position of the fused silica tube in the desolvation chamber, the skimmer size, and the ion-source temperature. (It is noted that both the sensitivity and ionization softness can be influenced dramatically by changing the position of the fused silica tube in the desolvation 
chamber independently. The optimization scheme described in this paper was preceded by optimization of the position of the fused silica in the desolvation chamber.) Consequently, only five variables, namely, the acetonitrile $(\mathrm{ACN})$ mobile phase composition, liquid chromatography flow rate, nebulizer-flow pressure, desolvation-chamber temperature, and the ammonium-acetate concentration in the mobile phase were evaluated for their effects on the Hewlett-Packard particle-beam system.

\section{Experimental}

A Hewlett-Packard (Avondale, PA) particle-beam interface was custom built into the ion source housing of the Finnigan 4500 gas chromatography-mass spectrometry (GC/MS) system (Finnigan-MAT, San Jose, $\mathrm{CA}$ ) in our laboratory. It was attached through the right vacuum port so that the gas chromatography
(GC) column could remain coupled with the ion source through the left vacuum port. A Waters 600-MS liquid chromatograph (Milford, MA) equipped with a variable UV detector was employed to pump the LC effluents into the particle beam interface. 4-Methoxy1-phenyl acetic acid (PMPA; MW =166) was used as a model compound for the optimization study of the Hewlett-Packard particle-beam interface for the following reasons. First, experience tells us that our particle-beam interface works very well for aromatic compounds. Second, PMPA is a thermally labile compound that tails badly on a nonpolar GC column. Finally, a large number of aromatic compounds have been determined in our laboratory. Owing to a high relative standard deviation observed at a low level of analyte (i.e., less than $1 \mu \mathrm{g}$ ), approximately $2 \mu \mathrm{g}$ of PMPA was injected into the particle-beam interface for this study. No signal saturation was observed under designed experimental conditions. The retention time,

Table 1. Particle-beam LC/MS interface experiment worksheet and results

\begin{tabular}{|c|c|c|c|c|c|c|}
\hline $\begin{array}{c}\text { Solvent } \\
(\%)\end{array}$ & $\begin{array}{c}\text { Nebulizer } \\
\text { flow } \\
\left(\mid b_{g} / \text { in. }{ }^{2}\right)\end{array}$ & $\begin{array}{c}\text { Desolvation } \\
\text { Temp. } \\
\left({ }^{\circ} \mathrm{C}\right)\end{array}$ & $\begin{array}{c}\text { Salt } \\
\text { (molar) }\end{array}$ & $\begin{array}{l}\text { Flow rate } \\
(\mathrm{mL} / \mathrm{min})\end{array}$ & $\begin{array}{c}\text { Peak area } \\
\text { (Detector counts) }\end{array}$ & $\begin{array}{c}\text { Softness } \\
\left(I_{m / 2166} / I_{m / 2121}\right)\end{array}$ \\
\hline 95.0 & 55.0 & 65.0 & 0.000 & 0.20 & 2,310430 & 0.17 \\
\hline 95.0 & 55.0 & 35.0 & 0.000 & 0.80 & $2,201,770$ & 0.18 \\
\hline 95.0 & 25.0 & 35.0 & 0.000 & 0.20 & 726,223 & 0.16 \\
\hline 5.0 & 55.0 & 35.0 & 0.000 & 0.20 & $2,040,210$ & 0.18 \\
\hline 5.0 & 25.0 & 35.0 & 0.000 & 0.80 & 10 & $1 e-04$ \\
\hline 50.0 & 40.0 & 50.0 & 0.000 & 0.50 & $3,364,830$ & 0.16 \\
\hline 5.0 & 25.0 & 65.0 & 0.000 & 0.20 & 171,075 & 0.08 \\
\hline 5.0 & 55.0 & 65.0 & 0.000 & 0.80 & 10 & $1 e-04$ \\
\hline 95.0 & 25.0 & 65.0 & 0.000 & 0.80 & $1,453,080$ & 0.14 \\
\hline 50.0 & 40.0 & 50.0 & 0.010 & 0.50 & $4,749,570$ & 0.20 \\
\hline 50.0 & 40.0 & 50.0 & 0.010 & 0.50 & $3,896,960$ & 0.18 \\
\hline 50.0 & 55.0 & 50.0 & 0.010 & 0.50 & $12,947,800$ & 0.19 \\
\hline 50.0 & 40.0 & 50.0 & 0.010 & 0.80 & $5,228,130$ & 0.18 \\
\hline 50.0 & 40.0 & 50.0 & 0.010 & 0.50 & $1,832,020$ & 0.19 \\
\hline 50.0 & 40.0 & 65.0 & 0.010 & 0.50 & $1,438,140$ & 0.19 \\
\hline 50.0 & 40.0 & 35.0 & 0.010 & 0.50 & $1,085,350$ & 0.15 \\
\hline 5.0 & 40.0 & 50.0 & 0.010 & 0.50 & 17.647 & 0.05 \\
\hline 50.0 & 25.0 & 50.0 & 0.010 & 0.50 & $2,722,770$ & 0.18 \\
\hline 50.0 & 40.0 & 50.0 & 0.010 & 0.50 & $4,181,950$ & 0.20 \\
\hline 50.0 & 40.0 & 50.0 & 0.010 & 0.20 & $2,166,430$ & 0.18 \\
\hline 50.0 & 40.0 & 50.0 & 0.010 & 0.50 & $3,471,610$ & 0.18 \\
\hline 50.0 & 40.0 & 50.0 & 0.010 & 0.50 & $4,045,180$ & 0.18 \\
\hline 95.0 & 40.0 & 50.0 & 0.010 & 0.50 & $4,228,900$ & 0.20 \\
\hline 50.0 & 40.0 & 50.0 & 0.020 & 0.50 & $5,156,340$ & 0.19 \\
\hline 95.0 & 25.0 & 65.0 & 0.020 & 0.20 & 298,456 & 0.13 \\
\hline 95.0 & 55.0 & 65.0 & 0.020 & 0.80 & $2,815,110$ & 0.18 \\
\hline 5.0 & 55.0 & 35.0 & 0.020 & 0.80 & 6,719 & 0.01 \\
\hline 5.0 & 25.0 & 35.0 & 0.020 & 0.20 & 642,480 & 0.17 \\
\hline 5.0 & 25.0 & 65.0 & 0.020 & 0.80 & 100,199 & 0.13 \\
\hline 95.0 & 55.0 & 35.0 & 0.020 & 0.20 & 11.571 .400 & 0.22 \\
\hline 95.0 & 25.0 & 35.0 & 0.020 & 0.80 & $2,202,260$ & 0.19 \\
\hline 5.0 & 55.0 & 65.0 & 0.020 & 0.20 & $1,968,450$ & 0.19 \\
\hline
\end{tabular}


measured from the onset peak appearance on the UV detector to that on the mass spectrometer detector, was approximately $20 \mathrm{~s}$ and remained nearly unchanged under the experimental conditions. The peak width and symmetry registered on the mass spectrometer scope were nearly identical to those on the UV chart recorder in the optimum region. The sensitivity was measured by integration across the reconstructed total ion current (TIC), and the ionization softness was measured by calculating the ratio of averaged peak heights of the molecular ion of PMPA (of $\mathrm{m} / \mathrm{z} 166$ ) to that of its major fragment ion at $m / z 121$. Peak heights were averaged from several scans across the TIC peak. The sensitivity of the mass spectrometer was checked and adjusted between experiments. The ion-source temperature was held at $250^{\circ} \mathrm{C}$ throughout this work. Although the ion-source temperature was found to have a significant effect on the sensitivity of the particle beam, an ion-source temperature of $250^{\circ} \mathrm{C}$ was the highest temperature achievable with the Finnigan 4500 mass spectrometer. A high ion-source temperature is believed to assist desorption from the particle beam
Table 2. Least squares coefficients for $\log _{e}(\text { area })^{\mathrm{a}}$

\begin{tabular}{lrcrc}
\hline \multicolumn{1}{c}{ Term } & \multicolumn{1}{c}{ Coeff. } & Std. Error & T-value & Signif. \\
\hline \hline Constant & 15.0216 & 0.2664 & 56.3907 & 0.0001 \\
Solvent & 2.2984 & 0.2349 & 9.7832 & 0.0001 \\
Nebulizer flow & 0.4406 & 0.2349 & 1.8756 & 0.0754 \\
Desolvation temp. & -0.0581 & 0.2349 & -0.2473 & 0.8072 \\
Salt conc. & 1.0456 & 0.2349 & 4.4505 & 0.0002 \\
Flow rate & -1.5222 & 0.2349 & -6.4795 & 0.0001 \\
Solvent**2 & -2.8171 & 0.3552 & -7.9314 & 0.0001 \\
Solvent* salt conc. & -0.9767 & 0.2492 & -3.9195 & 0.0008 \\
Solvent* flow rate & 1.9214 & 0.2492 & 7.7108 & 0.0001 \\
Neb. flow* desolv. & -0.9060 & 0.2492 & -3.6358 & 0.0016 \\
Temp & & & & \\
Neb. flow* flow rate & -0.6534 & 0.2492 & -2.6222 & 0.0163 \\
Salt conc.* flow rate & 0.8984 & 0.2492 & 3.6055 & 0.0018 \\
\hline
\end{tabular}

No. cases $=32$; condition no. $=2.646 ; R^{2}=0.9432 ; R_{\mathrm{adj}}^{2}=$ 0.9119 ; residual degrees of freedom $=20 ;$ Root-mean-square error $=0.9967$.

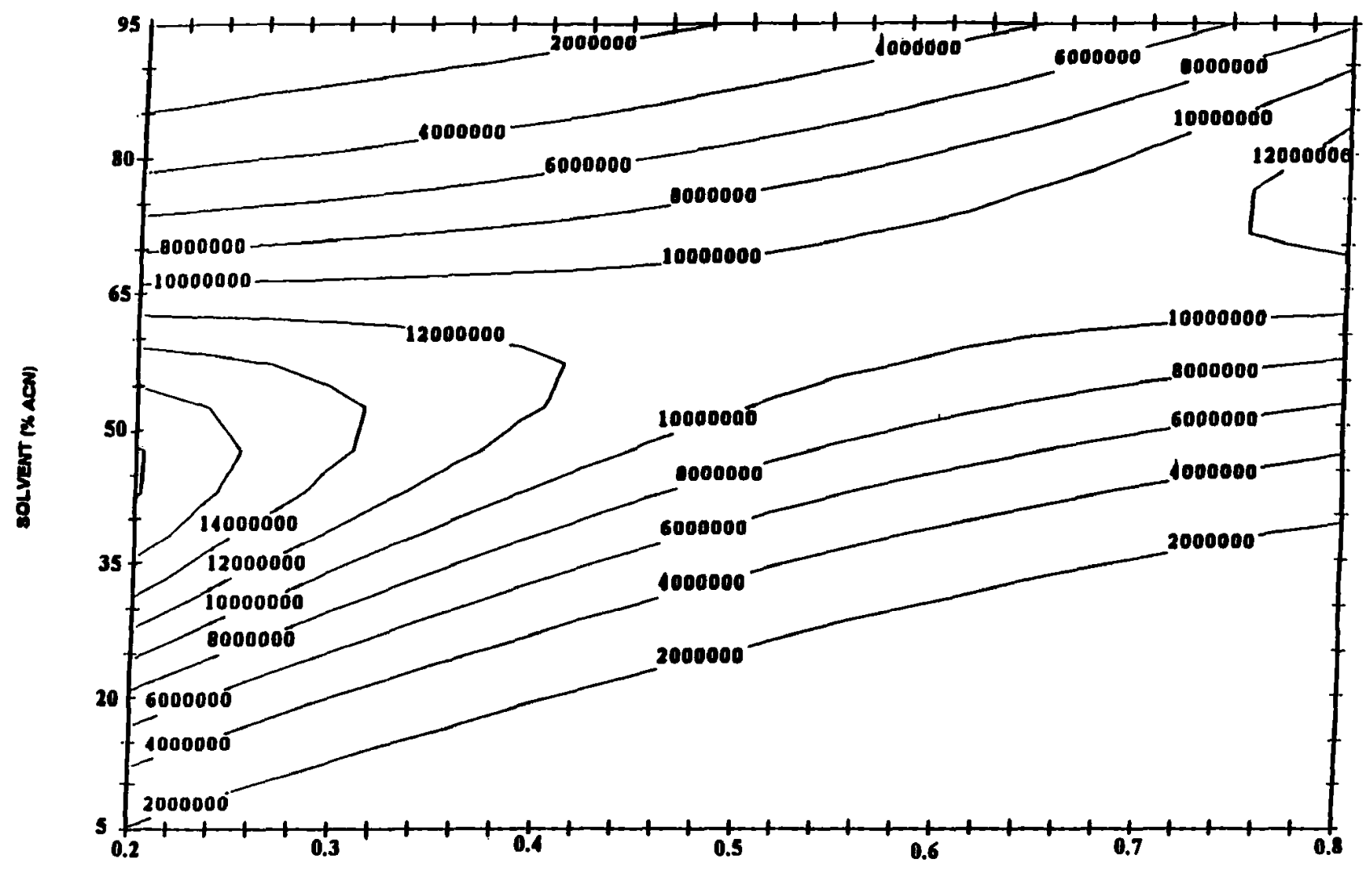

Frow anTe (mumin)

Figure 1. The contour plot of sensitivity as a function of both organic solvent composition and LC flow rate as a result of response surface optimization. The nebulizer-flow pressure was set at 40 $\mathrm{lb} /$ in. $^{2}$ and ammonium acetate concentration was set at $0.02 \mathrm{~F}$, while the desolvation chamber temperature was set at $50^{\circ} \mathrm{C}$. 
and/or to break up the particle beam into a molecular beam. Higher temperatures also have higher sensitivity and minimal fragmentation in the chemical ionization mode.

The experiments for the statistical design were based on a response surface model (RSM) that used a central composite face (CCF) design $[4,5]$ with centerpoints to allow for the examination of the main effects, interactions, and some of the quadratic terms. RSM is a collection of mathematical and statistical techniques that are used to optimize a response of interest that is influenced by several variables. Because the relationship between the response and variables is unknown, an approximation for the true functional relationship between the response and variables is assumed. Usually, a lower order polynomial in some region of the independent variables is employed. If the response is well modeled by a linear function of the variables, then the approximating function is the first-order model. If there is curvature in the system, then a polynomial of higher degree must be used. There were 32 data points (Table 1) included in the model. The data were analyzed by using the computer program RS/Discover (BBN Software Products Corporation, 10 Fawcett Street, Cambridge, MA 02138) running on a
VAX 6410 computer (Digital Equipment Corp., Maynard, MA).

Both the $A C N$ and ammonium acetate were LC grade purity purchased from Fisher Scientific (Pittsburgh, PA) and used without further purification. PMPA was synthesized and purified in our laboratory. Because the purpose of the statistically designed experiments is to map the relationship among the variables and to determine optimum conditions, flow injections at different mobile phase compositions (i.e., simulating a gradient condition) without an LC column were conducted throughout the work. The optimum flow rate obtained by this process then determines the size of an LC column that should be used.

\section{Results and Discussion}

Thirty-two experimental runs were made according to the experimental design, and the results are shown in Table 1. Because the range of the data is large, all data were transformed into logarithms. The results fit a quadratic model very well, as indicated by the high $R^{2}$ and $R_{\text {adj }}^{2}$ (i.e., adjusted $R^{2}$ ) values and low condition number (i.e., 2.6) in Table 2. A condition number less than 10 in a model suggests that all the independent

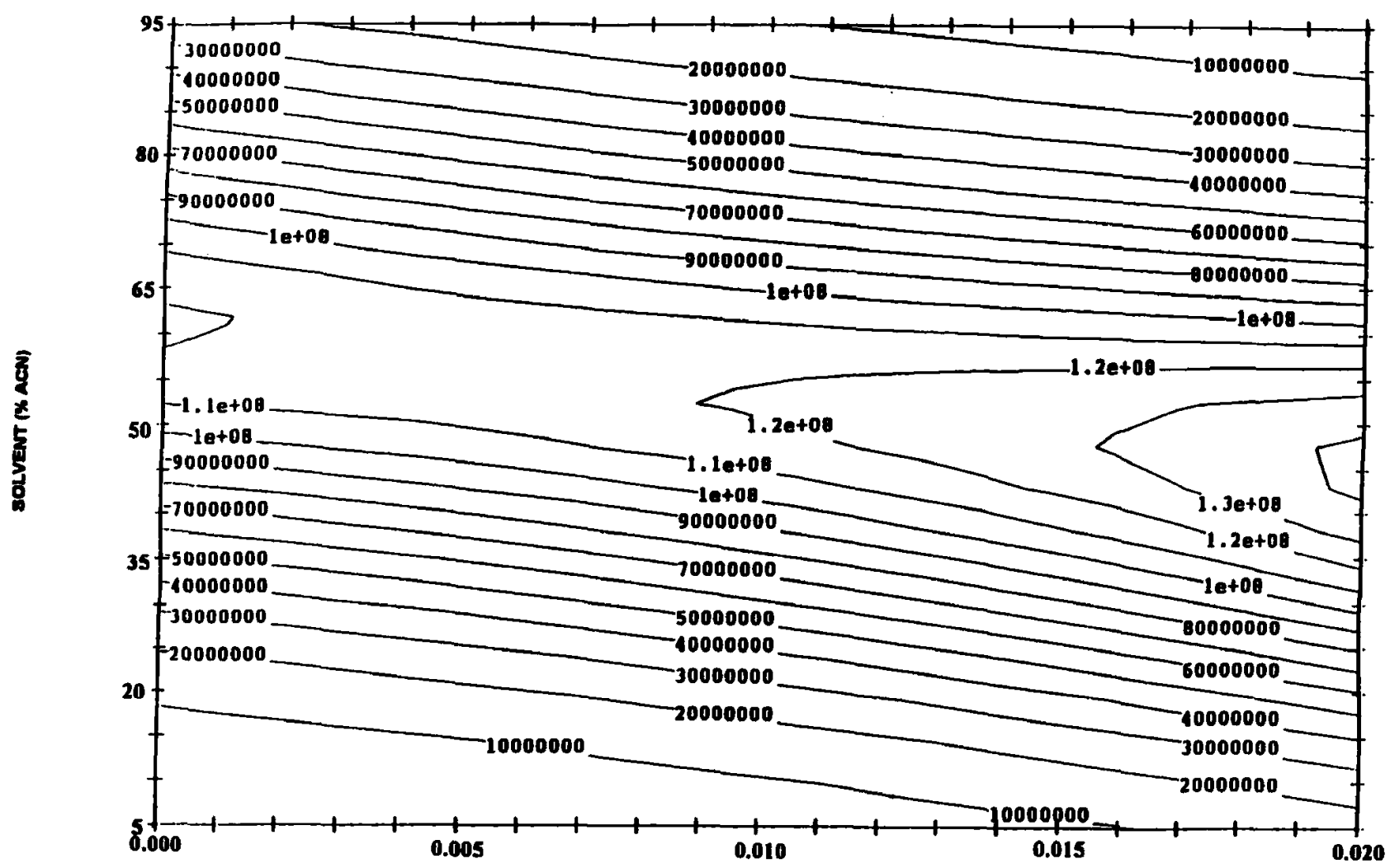

SALT (molin)

Figure 2. The contour plots of sensitivity as a function of both organic solvent composition and ammonium acetate concentration when the nebulizer-flow pressure, desolvation chamber temperature, and LC flow rate were set at $55 \mathrm{lb} / \mathrm{in}^{2}, 35^{\circ} \mathrm{C}$, and $0.2 \mathrm{~mL} / \mathrm{min}$, respectively. 
variables are not confounded. The model includes an important quadratic term in solvent and other interaction terms (Table 2). The main effects are solvent (\% $\mathrm{ACN}$ ), nebulizer-flow pressure, salt concentration, and the LC flow rate. The desolvation temperature is not an important standalone factor because its least squares coefficient is small and "signif" value is large (Table 2). This factor, however, is shown to couple with the nebulizer flow, as indicated in the "signif" column of Table 2. The "signif" number of 0.0016 means that the probability of no significant interaction of nebulizer flow and desolvation temperature is only $0.16 \%$. Figure 1 shows the contour plots that relate the sensitivity to the solvent composition and flow rate when the nebulizer-flow pressure, ammonium acetate concentration, and desolvation chamber temperature were set at $40 \mathrm{lb} /$ in. $^{2}, 0.02 \mathrm{~F}$, and $50^{\circ} \mathrm{C}$, respectively. The RSM optimization process involved study of the contour plots by variation of the values of the main factors. The optimal conditions for sensitivity were found to occur at a flow rate of $0.2 \mathrm{~mL} / \mathrm{min}, 45 \% \mathrm{ACN}, 55-\mathrm{lb} / \mathrm{in}^{2}{ }^{2}$ nebulizer pressure, and $35^{\circ} \mathrm{C}$ desolvation temperature in the presence of $0.02 \mathrm{~F}$ of ammonium acetate, as shown in Figure 2. Note that although there is also an optimized surface in the absence of ammonium acetate and $60 \%$ ACN (Figure 2), this region is much smaller than the wider region to the right. The three-dimensional plot of the response surface and the optimized parameters for the sensitivity are shown in Figure 3. The optimal conditions for softness follow nearly the same trend. The resulting optimal response surfaces

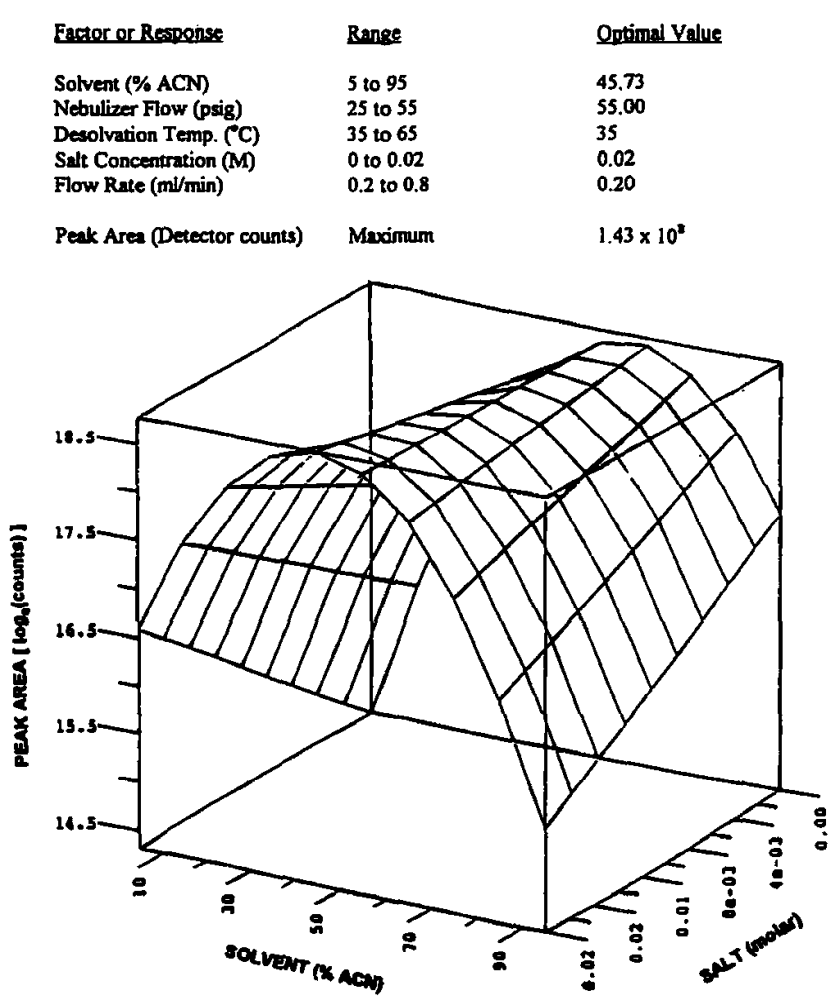

Figure 3. The optimized parameters and three-dimensional plot for sensitivity as a result of response surface optimization. and optimized parameters for the ionization softness are shown in Figure 4.

The present results indicate that there are significant differences between the Hewlett-Packard particlebeam and the Extrel Thermabeam interface [7]. Although both the peak area and ionization softness of the Hewlett-Packard particle-beam interface are optimized at low flow rates and about $50 \%$ organic modifier, the peak area and ionization softness of the Extrel Thermabeam interface are optimized at low and high percentages of organic modifier, respectively [7]. The addition of an ammonium salt to the eluant also has a significant influence on the performance of the Hewlett-Packard particle-beam, but not on the Extrel Thermabeam interface. [This material was presented at the 40th ASMS Spectrometry, held in Washington DC, May 31-June 5, 1992. From discussions with other particle-beam users, it was concluded that the addition of ammonium acetate to Vestec's universal interface (Vestec Corp., Houston, TX) had no effëct on sensitivity, but was observed to cause a slight increase in sensitivity on Extrel's Thermabeam. Hewlett-Packard's particle beam was found to exhibit the greatest increase in sensitivity by the addition of ammonium acetate.] Last, the Hewlett-Packard particle beam appears to yield very reliable and reproducible results on the basis of the quality of the model fitting. In contrast, the poorer fit of the data [7] from Extrel's Thermabeam experimental design to the model for this interface

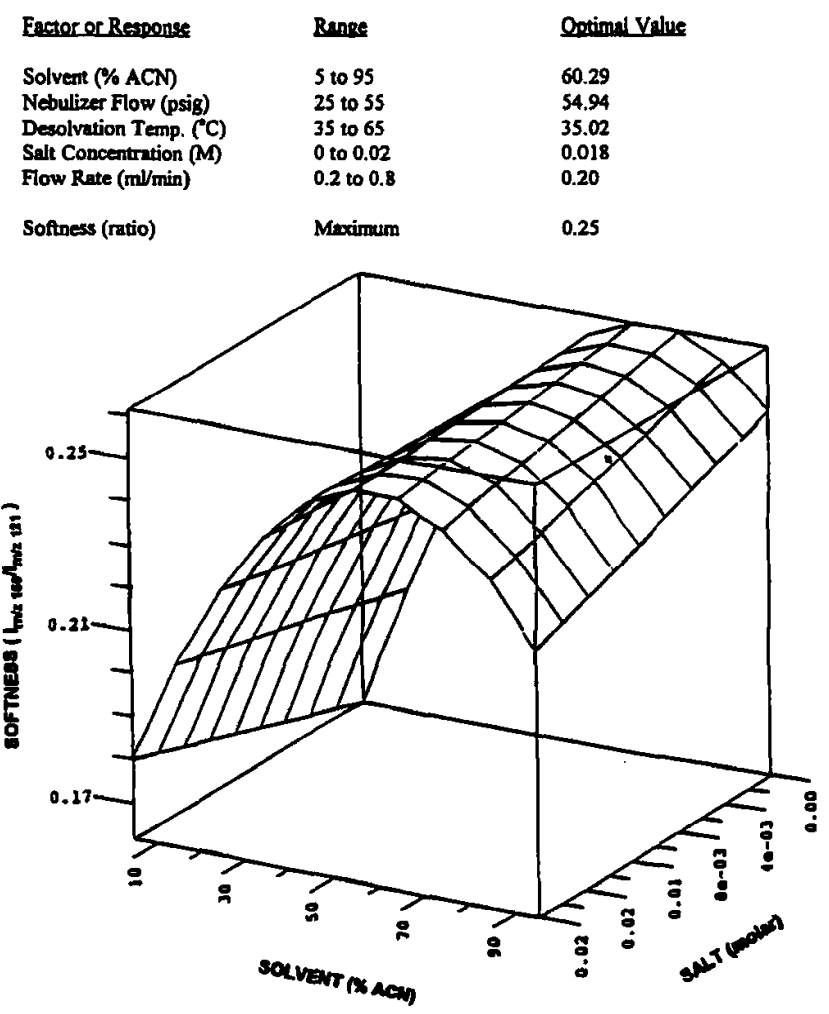

Figure 4. The optimized parameters and three-dimensional plot for ionization softness as a result of response surface optimization. 
suggests that either the interface gives data with high variability or some important factors have been left out of the experimental design. For instance, keeping the LC flow rate at $0.4 \mathrm{~mL} / \mathrm{min}$, which is recommended by both Hewlett-Packard and Extrel, in the Extrel experimental design [7] is probably a mistake. LC flow rate and percentage of the organic modifier are shown to be the most important factors in optimization of the Hewlett-Packard particle beam interface, that is, large least squares coefficients in Table 2. The observed effect of ammonium acetate on the optimization may be associated with its ability to "salt out" the water or make smaller aerosol particle sizes.

Unlike Extrel's Thermabeam interface, the biggest drawback for Hewlett-Packard's particle-beam interface is that both the sensitivity and softness decline when the mobile phase has a high percentage of water or organic modifier.

Although the optimization process was modeled by the injection of a single compound, (PMPA), it is found that the optimal regime for different aromatic compounds (up to molecular weight of 550) is nearly unchanged with regard to the peak shape and peak area. This was established by adjustment of the parameter settings away from the optimal region shown in Figures 3 and 4 . It is likely that different classes of compounds may be optimized under different conditions due to their different transport efficiency (Huang, S. K.; Garcia, D. M., unpublished results) [8]. Dependent on the volatility of the analytes, compounds that are too volatile or have too low molecular weight (e.g., 4-hydroxyacetophenone) suffer a great transport loss as determined by comparison of the mass spectrometer response with the UV trace. On the other hand, low volatility compounds such as 1,1,1-(4-hydroxyphenyl) ethane suffer the disadvantage of plugged skimmers in the momentum separator after even three injections (Huang, S. K.; Garcia, D. M., unpublished results). [Since Extrel uses stainless skimmers in their Thermabeam interface, it is far less prone to plugging problems than $\mathrm{HP}^{\prime}$ 's particle beam design for 1,1,1-(4hydroxyphenyl) ethane.] Nevertheless, these observations do not contrast with those used to obtain the optimum conditions previously described. In practice, our experience suggests that the particle-beam interface works well on aromatic or cyclic compounds [9] with moderate volatility (i.e., moderate molecular weights and thermal lability typically). Some high molecular weight compounds that have moderate volatility, such as 4,4-bis[(3',5'-di-t-butyl-4'-hydroxy- phenylprionoxymethyl)]-1,7-bis[( $3^{\prime \prime}, 5^{\prime \prime}$-di-t-butyl-4"-hydroxyphenylmethyl)] haptanoic acid ester (Huang, S. K.; Garcia, D. M., unpublished results) or Irganox 1010 (MW $=1176$ ), were successfully characterized by the optimized particle-beam LC/MS technique.

\section{Conclusions}

The best conditions for both sensitivity and ionization softness for the Hewlett-Packard particle-beam LC/MS interface occur at $55-\mathrm{lb} / \mathrm{in}^{2}$ nebulizer flow, $35^{\circ} \mathrm{C}$ desolvation chamber temperature, with approximately $45 \%$ organic modifier in the presence of $0.02 \mathrm{~F}$ ammonium acetate, and an LC flow rate of $0.2 \mathrm{~mL} / \mathrm{min}$. Hewlett-Packard's particle-beam interface is capable of production of reliable data, but cannot accommodate a mobile phase with high concentrations (greater than $80 \%$ ) of water or organic solvent.

We have successfully employed these optimized conditions to analyze a large number of pharmaceutical intermediates in our corporation. Therefore, we think that the optimization by virtue of statistical design is far superior to the COST (change one variable at a specific time) approach.

\section{Acknowledgment}

The encouragement of the Hoechst Celanese Publication Committee to publish this article and their critical review of this paper are greatly appreciated.

\section{References}

1. Willoughby, R. C.; Browner, R. F. Anal. Chem. 1984, 56, 2626-2631.

2. Winker, P. C.; Perkins, D. D.; Williams, W. K.; Browner, R. F. Anal. Chem. 1988, 60, 489-493.

3. Behymer, T. D.; Bellar, T. A.; Budde, W. L. Anal. Chem. 1990, 62, 1686-1690.

4. Box, G. E. P.; Hunter, W. G.; Hunter, S. J. Statistics for Experimenters; Wiley: New York, 1978.

5. Montgomery, D. C. Design and Analysis of Experiments; Wiley: New York, 1991.

6. Mark, H; Workman, J., Jr., Spectroscopy, 1994, 9, 26-27; 9, 30-32; 10, 17-20.

7. Huang, S. K.; Glancy, S. B. 39th ASMS Conference on Mass Spectrometry and Allied Topics; Nashville, TN, May 19-24, 1991; Huang, S. K.; Glancy, S. B.; Stansbury, W. F. Rapid Commun. Mass Spectrom. 1993, 7, 722-724.

8. Wilkes, J. G.; Leonidou, I.; Vestal, M. L., the 40th ASMS Conference on Mass Spectrometry and Allied Topics, Washington, DC, May 31-June 5, 1992.

9. Kuila, D.; Huang, S. Org. Mass Spectrom. 1994, 29, 226-231. 